\title{
Diagnosis and differential diagnosis of Asperger syndrome
}

\author{
Michael Fitzgerald \& Aiden Corvin
}

Asperger syndrome is an uncommon condition, but probably more common than classic autism (the only published population study estimated prevalence at 36 per 10000 children for Asperger syndrome and 5 per 10000 for autism (Ehlers \& Gillberg, 1993)). Misdiagnosis or delayed diagnosis of this disorder is a serious problem, and the average age at diagnosis is several years later than for autism (Gillberg, 1989). Obviously, this can be traumatic for individuals and families; furthermore, the most effective intervention programmes begin early, and establishing management strategies at an early age can minimise later behavioural problems (Howlin, 1998).

In 1944 Hans Asperger described a condition he termed autistic psychopathy, characterised by problems in social integration and non-verbal communication associated with idiosyncratic verbal communication and an egocentric preoccupation with unusual and circumscribed interests. Patients with this condition had difficulties with empathy and intuition and had a tendency to intellectualisation. They were also clumsy (50-90\% had motor coordination problems), found it hard to take part in team sports and exhibited behavioural difficulties including aggression and being victims of bullying. Asperger did not provide diagnostic criteria for this condition and it remained obscure until a review article by Lorna Wing in 1981.

Wing renewed interest in the condition, which she renamed Asperger syndrome, and described the following difficulties in the first 2 years of life of children with the condition:

(a) a lack of normal interest and pleasure in people around them; (b) a reduction in the quality and quantity of babbling;

(c) a significant reduction in shared interests;

(d) a significant reduction in the wish to communicate verbally or non-verbally;

(e) a delay in speech acquisition and impoverishment of content;

(f) no imaginative play or imaginative play confined to one or two rigid patterns.

A number of authors have subsequently suggested diagnostic criteria, but the six proposed by Gillberg (1991) are, arguably, closest to Asperger's original description of the syndrome (Box 1). Inclusion of the syndrome in both international diagnostic systems (ICD-10 (World Health Organization, 1992) and DSM-IV (American Psychiatric Association, 1994)) has resulted in broad clinical recognition of the diagnosis, but also in confusion. Asperger syndrome has been renamed Asperger disorder in DSM-IV, and the criteria of both differ from Gillberg's criteria and Asperger's original description of the syndrome.

Box 1 Gillberg's (1991) diagnostic criteria for Asperger syndrome

Social impairments

Narrow interests

Repetitive routines

Speech and language peculiarities

Non-verbal communication problems

Motor clumsiness

Michael Fitzgerald is Henry Marsh Professor of Child Psychiatry at Trinity College Dublin (Child and Family Centre, Ballyfermot Road, Ballyfermot, Dublin 10, Ireland. Tel: +353 1626 7512; fax: +353 1454 4418; e-mail: Fitzi@iol.ie). His primary research interests are autism and autistic spectrum disorders. Aiden Corvin is a Wellcome Trust Research Fellow in Mental Health at the Department of Psychiatry at Trinity Centre for Health Sciences, St. James's Hospital, Dublin. Dr Corvin was formerly a registrar in child psychiatry at the Child and Family Centre, Ballyfermot. His research interests include autism spectrum disorders and psychiatric genetics, particularly of psychotic disorders. 
Box 2 DSM-IV criteria for the diagnosis of Asperger disorder

A. Qualitative impairment in social interaction, as manifested by at least two of the following:

(1) marked impairment in the use of multiple nonverbal behaviors such as eye-to-eye gaze, facial expression, body postures, and gestures to regulate social interaction

(2) failure to develop peer relationships appropriate to developmental level

(3) a lack of spontaneous seeking to share enjoyment, interests, or achievements with other people (e.g., by a lack of showing, bringing, or pointing out objects of interest to other people)

(4) lack of social or emotional reciprocity

B. Restricted repetitive and stereotyped patterns of behavior, interests, and activities, as manifested by at least one of the following:

(1) an encompassing preoccupation with one or more stereotyped and restricted patterns of interest that is abnormal either in intensity or focus

(2) apparently inflexible adherence to specific, nonfunctional routines or rituals

(3) stereotyped and repetitive motor mannerisms (e.g., hand or finger flapping or twisting, or complex whole-body movements)

(4) persistent preoccupation with parts of objects

(American Psychiatric Association, 1994: p. 77)

This disturbance must be clinically significant, but without clinically significant language delay or delay in cognitive development or other skills

The DSM-IV diagnosis is based on impairment of social interaction and the presence of stereotypical or repetitive behaviours (Box 2). Diagnosis requires that the impairment is clinically significant, occurs before 3 years of age and excludes clinically significant delay in language, cognition or other skills. The ICD-10 research criteria (World Health Organization, 1993) are virtually identical. By excluding speech and language difficulties, the DSM definition of Asperger disorder is narrower than Asperger syndrome as defined by Wing or Gillberg and would exclude some of the original cases described by Hans Asperger. As the vast majority of persons with Asperger disorder/syndrome do have speech and language abnormalities it was hoped that future text revisions of DSM-IV might correct this anomaly. Indeed, the recent DSMIV-TR guides that the occurrence of "no clinically significant delays in language does not imply that individuals with Asperger Disorder have no problems with communication" (American Psychiatric Association, 2000, p. 80). Examples given include unusual verbosity or subtle abnormalities of social communication (such as turn-taking in conversation). We feel that DSM-IV-TR draws attention to the issue, but underestimates the language difficulty involved. This paper examines the differential diagnosis of Asperger syndrome (Wing, 1981) and disorder (American Psychiatric Association, 1994), beginning with the more common and finish with the less common causes of diagnostic confusion.

\section{Differential diagnosis}

\section{Other pervasive developmental disorders (PDD)}

Autism/autism spectrum disorders (DSM-IV)

Autism shares the same DSM criteria for abnormal social interaction and behaviour as Asperger disorder, but requires additional impairments in communication (Box 3). Delay or impairment in social interaction, communication or behaviour must arise before age 3 years. In DSM-IV, if both autism and Asperger disorder diagnoses can be made, the autism diagnosis takes precedence. Unlike Asperger disorder (which excludes individuals with delays in cognitive abilities or other skills), autism can occur at all levels of ability: the majority (70\%) of cases have associated learning disabilities and almost half have an IQ below 50 .

In a prevalence study of autism, Wing \& Gould (1979) identified a large number of children who failed to meet the diagnostic criteria for classic autism, but had a triad of impairments involving social interaction, communication and imagination, with additional repetitive stereotyped activities. This triad of symptoms, termed the 'autistic spectrum', was recognised at all levels of intelligence and is included in DSM-IV as 'pervasive developmental 
Box 3 Additional DSM-IV criteria for autism

Must meet criteria $A$ and $B$ in Box 1, but in addition:

Qualitative impairments in communication as manifested by at least one of the following:

(a) delay in, or total lack of, the development of spoken language (not accompanied by an attempt to compensate through alternative modes of communication such as gesture or mime)

(b) in individuals with adequate speech, marked impairment in the ability to initiate or sustain a conversation with others

(c) stereotyped and repetitive use of language or idiosyncratic language

(d) lack of varied, spontaneous make-believe play or social imitative play appropriate to developmental level

(American Psychiatric Association, 1994: p. 70)

A total of six items from the social, communication and behavioural criteria are required. Delay in at least one of these domains must occur before age 3 years

disorder not otherwise specified', and in ICD-10 as 'atypical autism', 'other pervasive developmental disorders' or 'pervasive developmental disorders, unspecified'. The estimated prevalence of autism spectrum disorders (autistic spectrum disorders) may be as high as 91 per 10000 .

At present it is not clear whether Asperger syndrome is distinct from the autistic spectrum disorders. Diagnostic uncertainty arises in patients of near normal cognitive ability (total IQ >70) with autistic spectrum disorders, who are described as having high-functioning autism (HFA). Differences are reported between Asperger syndrome and HFA, for example, verbal skills being significantly greater than non-verbal ones in Asperger syndrome - the opposite of the pattern reported in HFA. This differentiation may be simplistic, as it depends on the diagnostic system used. A sample defined using Wing or Gillberg criteria for Asperger syndrome would include children with abnormal or delayed language and, by definition, worse verbal skills than a DSM or ICD sample, which would exclude these individuals. Attempts to separate Asperger syndrome or HFA categorically based on presence or absence of language delay are artificial. Comparison studies have also failed to control adequately for IQ differences between samples in many cases. Current research data do not convincingly support the separation of Asperger syndrome and the autistic spectrum disorders as distinct disorders. Both Asperger syndrome and autistic spectrum disorders are about five times more common in boys than in girls; segregate within the same families; appear strongly genetic (American Psychiatric Association, 2000); and share similar comorbidity (Gillberg \& Billstedt, 2000). If autism and Asperger syndrome differ at all, it is in the degree of impairment rather than in having discrete, specific and independent features. This is supported by outcome studies; both are associated with social difficulties persisting into adulthood, but these are less severe in Asperger syndrome. Combining Asperger syndrome and autistic spectrum disorders into an autistic spectrum is a better way forward.

In addition to the diagnostic criteria mentioned, a number of assessment instruments are available or in development for use with people with Asperger syndrome. These include the Asperger Syndrome Screening Questionnaire (ASSQ; Ehlers et al, 1999) and the Pervasive Developmental Disorder Questionnaire (PDD-Q; Baron-Cohen et al, 1996). The ASSQ is designed for completion by parents or teachers to screen for Asperger syndrome and other high-functioning autism spectrum disorders in school-age children. The PDD-Q is an 18-item questionnaire for parents, which includes questions specifically designed to identify clinical characteristics of Asperger syndrome.

These instruments were all designed for screening purposes, not to differentiate Asperger syndrome from HFA, but to identify higher-functioning individuals within the autistic spectrum. Each requires further work to fulfil psychometric requirements for sensitivity, specificity, reliability and validity. Of existing instruments used for the diagnosis of autistic spectrum disorders, the Autism Diagnostic Interview - Revised (ADI-R; Lord et al, 1994) may be useful in assessing individuals for Asperger syndrome. This instrument consists of three scales corresponding to the social, communication and behavioural impairments and is based on parent report. The Autism Diagnostic Observational Schedule - Generic (ADOS-G; Lord et al, 1999), a supplementary standardised interview and observational assessment may also be helpful. Accurate diagnosis in younger children (under 2 
Box 4 Assessment of Asperger syndrome

Assessment should include:

A medical examination to exclude medical causes, e.g. sensory impairments

A laboratory workup (to exclude fragile-X syndrome, for example)

Psychiatric evaluation for comorbidity

Psychological assessment (including IQ assessment)

Speech and language assessment

years of age) is difficult, despite the neurodevelopmental nature of these disorders. The Checklist for Autism in Toddlers (CHAT; Baird et al, 2000) may prove useful in identifying children at risk. Howlin (2000) provides a more extensive appraisal of available assessment instruments. Full assessment of Asperger syndrome requires a multi-disciplinary approach, as outlined in Box 4.

Pervasive developmental disorder not otherwise specified (DSM-IV)

Another diagnosis of relevance is pervasive developmental disorder not otherwise specified (PDDNOS). This is characterised by "a severe and pervasive impairment in the development of reciprocal social interaction or verbal and nonverbal communication skills or when stereotyped behaviour, interests and activities are present" (American Psychiatric Association, 1994: p. 77). This category in DSM-IV needed revision as the inclusion of the word 'or' twice greatly diluted its meaning and grossly widened it as a category. DSMIV-TR has corrected this error, requiring there to be an impairment in reciprocal social interaction associated with an impairment in communication skills or with stereotyped behaviour, interests or activities. In the past, particularly in the USA, PDDNOS was used as a synonym for Asperger syndrome, although for parents this title is unhelpful and confusing.

Other pervasive developmental disorders (DSM-IV)

Neither Rett disorder nor childhood disintegrative disorder are part of the autistic spectrum, and they are unlikely to represent sources of diagnostic confusion. Rett disorder is most common in girls and is characterised by apparently normal development in the first 5 months of life, with subsequent deceleration of head growth, loss of previously acquired hand skills, loss of social engagement, poorly coordinated gait and language problems (American Psychiatric Association, 2000). Similarly, childhood disintegrative disorder presents with loss of language, motor skills and bowel and bladder control following a 2-year history of normal development (American Psychiatric Association, 2000). In each case the diagnosis should be excluded by a detailed developmental history and physical examination.

\section{Schizophrenia spectrum disorders}

\section{Schizophrenia (DSM-IV)}

Schizophrenia is a disorder in which psychotic symptoms (delusions or hallucinations), thought disorder and so called 'negative symptoms' cause social and/or occupational dysfunction over time. Because individuals with Asperger syndrome have normal cognitive ability, restrictive behaviours and impairments in social interaction and communication can be misinterpreted as evidence of schizophrenia. People with Asperger syndrome have difficulty understanding the subtleties of social behaviour, but this should not be confused with evidence of psychotic disorder. In a clinical setting, asking individuals with Asperger syndrome whether they hear voices may induce a positive response, and they might concur that they hear voices "when people aren't there", but they may be refering to the voices of people in an adjacent room. Deficiencies in concrete thinking and in understanding how other minds think may cause patients with Asperger syndrome to misinterpret what is said to them, and they might as a result be labelled paranoid. Misinterpreting social contacts can also lead to inappropriate emotional responses, contributing to this impression. Persons with Asperger syndrome sometimes speak their thoughts out loud, which again can be misinterpreted by a psychiatrist.

Language abnormalities associated with autistic spectrum disorders include substitutions, literalness, problems with prosody, staccato speech and monotonous speech that is excessively pedantic and focused on details or obsessive questions. A tendency to direct the conversation towards obsessions could easily be mistaken for evidence of associative loosening. A comparison of thought disorder and affective flattening in patients with autism and with schizophrenia found that they did not differ in terms of affective flattening, and that adult patients with autism showed poverty of speech, poverty of content and perseveration (Ramsey et al, 1986). The autism group showed significantly less derailment and illogicality, suggesting that they would be unlikely to meet DSM or ICD criteria for thought disorder in schizophrenia. 
Social and communication deficits can be interpreted as evidence of negative symptomatology, so it is important when assessing functioning to establish premorbid ability. These conditions obviously differ in age at onset, developmental history and mental state examination. In DSM-IV, pervasive developmental disorder is an exclusion condition for schizophrenia and it should be suspected in atypical or non-responsive cases. Schizophrenia can co-occur in autistic spectrum disorders, but the additional diagnosis is made only if prominent delusions or hallucinations are present for at least 1 month (less with treatment). Despite an absence of epidemiological studies of psychiatric comorbidity in autistic spectrum disorders, it has been suggested that delusions or auditory hallucinations may be more common than in the general population, but the prevalence of schizophrenia (at $0.6 \%$ ) is comparable to general population levels.

Bleuler (1911), founder of the modern concept of schizophrenia, described four primary symptoms necessary for the disorder (the four As): ambivalence, loosening of associations, disturbance of affect and autism, which he defined as dependence on an internal unrealistic world. Both he and Kraepelin (1919) defined subgroups with social withdrawal and affective flattening, 'oddness' and 'eccentricity', being timid with a narrow circle of interests and cold relations to companions, and lacking sympathy or attachment. From these descriptions the concept of simple schizophrenia, considered by some to be a diagnostic waste-basket, entered the lexicon. The symptoms described are equally applicable to autistic spectrum disorders, and the subtype 'simple schizophrenia' has been removed from DSM-IV. Its retention in ICD-10 is a likely source of diagnostic confusion.

\section{Schizoid personality in childhood (DSM-IV)}

Schizoid personality in childhood is defined by solitariness, lack of empathy, emotional detachment, increased sensitivity, at times paranoid ideation, and single-minded pursuit of special interests. All these features are seen in Asperger syndrome, and comorbid issues (depression or behaviour problems in particular) are likewise similar for both conditions. On the basis of evidence presented in Wolff's (1998) discussion of schizoid personality in childhood, we have concluded that there is significant overlap between schizoid personality in childhood and Asperger syndrome.

\section{Schizotypal personality disorder (DSM-IV)}

The DSM-IV diagnosis of schizotypal personality disorder depends on odd beliefs or magical thinking, bizarre fantasies or preoccupations, odd thinking and speech, odd, eccentric or peculiar behaviour and appearance, lack of close friends and social anxiety. All of these criteria can also occur in Asperger syndrome, and Wolff (1998) regards "Asperger syndrome and schizoid/schizotypal disorders as interchangeable terms that identify roughly the same group of children". The conditions do differ in at least three important respects. First, there appears to be an increased rate of development of schizophrenia in schizotypal personality disorder. Second, schizotypal personality disorder and schizophrenia co-occur in families and appear genetically related. Third, prospective research of children at high risk of schizophrenia (Erlenmeyer-Kimling et al, 2000) suggests that some individuals later diagnosed with schizotypal personality disorder developed without impairments in reciprocal social interaction and communication.

\section{Attention-deficit hyperactivity disorder (DSM-IV)}

Attention-deficit hyperactivity disorder (ADHD) presents with inattention, distractability, fidgetiness, impulsivity and hyperactivity. Persons with HFA spectrum disorders may be hyperactive, impulsive, have a short attention span and share similar executive function deficits as patients with ADHD. The conditions differ in that ADHD lacks the classic impairment in reciprocal social interaction, narrow interests, repetitive routines and non-verbal problems of Asperger syndrome. In accordance with a hierarchical rule in DSM-IV, a person meeting the criteria for a pervasive developmental disorder cannot be diagnosed as having ADHD. This is not the case in ICD-10, in which a dual diagnosis of Asperger syndrome and ADHD is possible.

Gillberg \& Ehlers (1998) point out that children who meet criteria for ADHD may also meet the full criteria for Asperger syndrome. They mention one study, in which $21 \%$ of children with severe ADHD met the full criteria for Asperger syndrome and 36\% showed autistic traits. A developmental history is usually sufficient to separate ADHD from Asperger syndrome, but ADHD can present as soon as the child can walk, and it is important to consider that impulsivity can interfere with social relationships, making children appear unempathic. Indeed, children with ADHD can be so easily distracted that they appear to be in a world of their own and therefore seem socially disconnected. It is not surprising, therefore, that children with Asperger syndrome are not uncommonly misdiagnosed as having ADHD, since it is often the attention and hyperactive problems that parents first observe. 


\section{Obsessive compulsive disorders (DSM-IV)}

The core features of obsessive-compulsive disorder (OCD) are recurrent and persistent thoughts, impulses or images that are experienced at some time during the disturbance as intrusive and inappropriate and that cause marked anxiety or distress. The individual recognises that these are a product of his or her own mind. Compulsions involve repetitive behaviours or mental acts that a person feels driven to perform to reduce stress associated with some dreaded event or situation. An adult can recognise that they are excessive or unreasonable, but children cannot (American Psychiatric Association, 1994).

These phenomena, including the urge to count and manipulate numbers, to repeat the same action over and over, are similar to the repetitive routines associated with Asperger syndrome. Individuals with both conditions display ritualistic behaviour and resistance to change. Where they differ is that persons with Asperger syndrome have obsessive interests that are not experienced as ego-dystonic and, indeed, are often enjoyed. Baron-Cohen (1989) was critical of the use of the term obsession in persons with autism because the subjective phenomena of resistance to repetitive activities could not be discerned in autism. He suggested instead the phrase 'repetitive activities'. OCD generally has a much later onset and lacks the poor social emotional reciprocity, empathy problems and social skills difficulties of people with Asperger syndrome (Szatmari, 1998). Detailed analysis of current symptoms and an early developmental history are the key to making a correct diagnosis.

\section{Affective disorders}

Despite some overlap in symptomatology (including social withdrawal, lack of emotional response and loss of interest in relationships), affective disorders differ in representing a distinct change from premorbid functioning, and typically are associated with onset in adulthood. Epidemiological studies of psychiatric comorbidity are lacking in individuals with autistic spectrum disorders, but depression, anxiety disorders and bipolar disorder occur more commonly than in the general population and represent substantial morbidity (Gillberg \& Billstedt, 2000).

\section{Other diagnostic categories}

Many other overlapping categories are unrepresented in DSM-IV or ICD-10 and may be a source of confusion for families and professionals alike. Terms such as semantic pragmatic disorder, non-verbal learning difficulty and developmental learning disability of the right hemisphere have arisen as different specialities have struggled independently to categorise individuals with social disabilities who do not meet criteria for classic autism.

\section{Semantic pragmatic disorder}

Semantic pragmatic disorder (Rapin \& Allen, 1983) is probably not an uncommon cause of misdiagnosis. Children with autistic spectrum disorders often have some language difficulties and many will attend a speech therapist in the first instance and receive a diagnosis of semantic pragmatic disorder. This is characterised by "near-normal vocabulary, grammar, and phonology, but language use is abnormal in content and function and comprehension is also impaired. There are considerable difficulties in initiating or sustaining a conversation, making cohesive links in conversation from topic to topic, and words are used out of context" (Szatmari, 1998: p. 71). Is this an exact description of the language problems of Asperger syndrome (Wing, 1981)? These describe pragmatic language difficulties. Wing's criterion for a reduction in quality and quantity of babbling refers to expressive language difficulties; a delay in speech acquisition and impoverishment of content are receptiveexpressive language problems; and defective imaginative play is a receptive-pragmatic language difficulty. The definition of semantic pragmatic disorder includes no reference to problems of social and emotional interaction or to repetitive and stereotyped patterns of behaviour. The existence of semantic pragmatic disorder as a separate entity with clinical validity is questionable.

\section{Deficits in attention, motor control and perception}

The core features of deficits in attention, motor control and perception (DAMP; Gillberg et al, 1982) include a cross-situational disturbance of attention, gross and fine motor dysfunction and perceptual dysfunctions not accounted for by associated mental retardation or cerebral palsy. DAMP and Asperger syndrome are similar and can occur together. Overlapping features include: the condition being more common in boys, perceptual problems, a failure to adjust volume and pitch of voice and motor clumsiness (although the latter is not recognised in the Asperger disorder criteria). Whether they represent the same population is uncertain, as attention difficulties are not part of the definition of either Asperger syndrome or disorder, and neither are associated with delay in cognitive development. 
DAMP can also have significant speech and language difficulties, e.g. articulation problems, hypotonia of the mouth and certain variants of stuttering. Heredity appears to play a much lesser role in DAMP than in Asperger syndrome. It would appear that criminality is more common at followup in persons with DAMP than in those with Asperger syndrome. Gillberg (1995) found that about half of adults with DAMP had significant and persistent problems that included criminal offences.

\section{Multidimensionally impaired disorder}

Criteria for multidimensionally impaired disorder (MDI; Kumra et al, 1998) include a poor ability to distinguish fantasy from reality, impairment in interpersonal skills and multiple deficits in processing information. Fitzgerald (1998) has argued that MDI should be categorised with autism or Asperger syndrome because of the overlapping symptomatology. Kumra et al (1998) consider MDI an atypical variant of childhood-onset schizophrenia, as they share a similar pattern of cytogenetic abnormalities, neuropsychological deficits, structural brain abnormalities, smooth-pursuit eyetracking abnormalities, premorbid history and elevated rates of schizophrenia spectrum disorder in first-degree relatives. The nosological status of MDI is uncertain, but we feel that the clinical diagnosis of Asperger syndrome offers far greater opportunities to engage with appropriate educational, psychological and psychiatric services (Fitzgerald, 1998).

\section{Multiple complex developmental disorder}

The defining characteristics of multiple complex developmental disorder (MCDD; Cohen et al, 1987) are shown in Box 5. Thought disorder and affective dysregulation are more characteristic of MCDD subjects, whereas problems in social interaction, communication and behavioural adjustment are more typical of subjects with autistic disorder. As the core features can also occur in Asperger syndrome its nosological status is uncertain.

\section{Cerebellar affective syndrome}

Cerebellar affective syndrome (Schmahmann \& Sherman, 1998) presents with impairment of executive functions such as planning, set shifting, abstract reasoning and working memory. It also includes difficulties with spatial cognition, including visuo-spatial organisation with disinhibited or inappropriate behaviour. It differs from Asperger syndrome in that it is a late-onset

\section{Box 5 Multiple complex developmental} disorder (Cohen et al, 1987)

\section{Defining characteristics}

Affective regulation problems

Impaired capacity for relating

Impaired cognitive processing in children

Disorganisation precipitated by changes in routine

Impairment in empathy

Comorbid anxiety or depression

condition. The persons so far studied with this condition range in age from 23 to 74 years. They presented with post-infectious cerebellitis, cerebellar tumours and strokes. Differential diagnosis is easily made on history-taking. This is a differential diagnosis that should be considered in older patients.

\section{Dyslogia}

The syndrome of dyslogia was described by Jordan (1972) as the inability to apply logic and common sense in decision-making. Individuals with this difficulty make decisions based on partial facts and have difficulty in integrating data into a working whole. They have social difficulties similar to those of individuals with Asperger syndrome, and dyslogia may simply describe the same population.

Developmental learning disability of the right hemisphere (social-emotional learning disorder)

This disorder (Denckla et al, 1983) could be seen as the product of a lack of communication between neurologists and psychiatrists since there is such an overlap between this condition and Asperger syndrome. Children with the condition have difficulty understanding social and emotional information.

\section{Non-verbal learning disability}

Non-verbal learning disability (Myklebust, 1975) is characterised by deficits in perception, coordination, socialisation, non-verbal problem-solving and understanding of humour, but well-developed rote memory. As many people with Asperger syndrome have this disability, a primary diagnosis of Asperger syndrome is often preferred and is certainly the most clinically useful. This is an example of excessive diagnostic splitting, although non-verbal learning disability can occur with other disorders. 


\section{Discussion}

Asperger syndrome can be misdiagnosed as a variety of conditions (Box 6) requiring contradictory treatments and having a range of outcomes. Misdiagnosis as schizophrenia leads to the prescribing of neuroleptics and an unnecessary risk of tardive dyskinesia; misdiagnosis as ADHD to the prescription of psychostimulants (e.g. methylphenidate), which can cause deterioration in behaviour in this population. The condition may also be at the root of treatment-resistant mental illness in adult psychiatry. Diagnostic confusion increases individual and family burden and causes families to seek unhelpful therapies or join the wrong support groups. Neuropsychiatric disorders may share similar symptoms, for example autistic behaviour in schizophrenia or hyperactivity in ADHD. The problems this poses clinicians are compounded by partial diagnostic assessments or the use of outdated diagnostic categorisations (e.g. putting all 'autistictype behaviours' into a psychotic category or being unaware of the existence of Asperger syndrome, which is not included in ICD-9 or DSM-III).

Clearly, the differentiation of Asperger disorder from other conditions is complex because of the many possibilities for misdiagnosis. The key to correct diagnosis is a precise early developmental history, with a systematic discussion of all the criteria set out for Asperger syndrome (Wing, 1981; Gillberg, 1991) or Asperger disorder (American Psychiatric Association, 1994). Assessment instruments such as the ADI-R may be useful in establishing diagnosis. A multi-disciplinary team approach is critical, and diagnosis from a solely neurological, speech and language or educational point of view must cease if families are to be spared confused partial diagnoses. Although higher-functioning autistic spectrum disorders and Asperger syndrome may describe the same population, the latter term remains useful. As applied to higher-functioning children it is more acceptable to parents and ensures appropriate service provision for a group who, despite relatively normal cognitive ability, may have comprehensive difficulties.

\section{References}

American Psychiatric Association (1994) Diagnostic and Statistical Manual of Mental Disorders (4th edn) (DSM-IV). Washington DC: APA.

- (2000) Diagnostic and Statistical Manual of Mental Disorders (4th edn, text revision) (DSM-IV-TR). Washington DC: APA

Asperger, H. (1944) Die "Autistischen Psychopathen" im Kindesalter. Archives für Psychiatrie und Nervenkrankheiten, 117, 76-136. Trans. (1991) in Autism and Asperger
Box 6 Differential diagnosis of Asperger syndrome

Other pervasive developmental disorders: Autism

Pervasive developmental disorder not otherwise specified

Childhood disintegrative disorder

Rett disorder

Schizophrenia spectrum disorders:

Schizophrenia

Schizotypal disorder

Schizoid personality disorder

Adult attention-deficit hyperactivity disorder

Obsessive-compulsive disorder

Depression

Other diagnostic categories:

Semantic pragmatic disorder

Deficits in attention, motor control and perception

Multidimensionally impaired disorder

Multiple complex developmental disorder

Cerebellar affective syndrome

Developmental learning disability of the right hemisphere (social-emotional learning disorder

Non-verbal learning disability

Syndrome (ed. U. Frith), pp. 37-92. Cambridge: Cambridge University Press.

Baird, G., Charman, T., Baron-Cohen, S., et al (2000) A screening instrument for autism at 18 months of age: a 6year follow-up study. Journal of the American Academy of Child and Adolescent Psychiatry, 39, 694-702.

Baron-Cohen, S. (1989) Do autistic children have obsessions and compulsions? British Journal of Clinical Psychology, 28, 193-200.

-, Cox A., Baird G., et al (1996) Psychological markers in the detection of autism in infancy in a large population. British Journal of Psychiatry, 168, 158-163.

Bleuler, E. (1911) Dementia Praecox or the Group of Schizophrenias. Trans. (1950) by G. Zinkin. New York: International University Press.

Cohen, D., Paul, R. \& Volkmar, F. (1987) Issues in classification of pervasive developmental disorders and associated conditions. In Handbook of Autism and Pervasive Developmental Disorders (eds D. J. Cohen, A. M. Donnellan \& R. Paul), pp. 5-40. New York: John Wiley \& Sons.

Denckla M. B.(1983) The neuropsychology of social-emotional learning disabilities. Archives of Neurology, 40, 461-462.

Ehlers, S. \& Gillberg, C. (1993) The epidemiology of Asperger syndrome. A total population study. Journal of Child Psychology and Psychiatry, 34, 1327-1350.

- _ — \& Wing, L. (1999) A screening questionnaire for Asperger syndrome and other high functioning autism spectrum disorders in school age children. Journal of Autism and Developmental Disorders, 29, 439-484.

Erlenmeyer-Kimling, L., Rock, D., Roberts, S. A., et al (2000) Attention, memory, and motor skills as childhood predictors of schizophrenia-related psychoses: the New York HighRisk Project. American Journal of Psychiatry, 157, 1416-1422. 
Fitzgerald, M. (1998) Multidimensionally impaired disorder. Journal of the American Academy of Child and Adolescent Psychiatry, 37, 1125-1126.

Gillberg, C. (1985) Clinical Child Neuropsychiatry. Cambridge: Cambridge University Press.

- (1989) Asperger syndrome in 23 Swedish children. Developmental Medicine and Child Neurology, 31, 520-531.

- (1991) Clinical and neurobiological aspects of Asperger's syndrome in six families studied. In Autism and Asperger's Syndrome (ed. U. Frith), pp. 122-146. Cambridge: Cambridge University Press.

— \& Billstedt, E. (2000) Autism and Asperger syndrome: coexistence with other clinical disorders. Acta Psychiatria Scandinavica, 102, 321-330.

- Rasmussen, T., Carlstrom, G., et al (1982) Perceptual, motor and attentional deficits in 6 year old children. Epidemiological aspects. Journal of Child Psychology and Psychiatry, 23, 131-144.

_ \& Ehlers, S. (1998) High functioning people with autism and Asperger's syndrome. In Asperger's Syndrome or High Functioning Autism (eds E. Schoplar, G. Mesihov \& L. J. Kunce), pp. 77-100. New York, NY: Planum.

Howlin, P. (1998) Practitioner review: psychological and educational treatments for autism. Journal of Child Psychology and Psychiatry, 39, 307-322.

- (2000) Assessment instruments for Asperger Syndrome. Child Psychology and Psychiatry Review, 5, 120-129.

Jordan, D. R. (1972) Dyslexia in the Classroom. Columbus, $\mathrm{OH}$ : Merril.

Kraepelin, E. (1919) Dementia Praecox and Paraphrenia. In Textbook of Psychiatry (8th edn), pp. 176-177. Edinburgh: E. S. Livingstone.

Kumra, S., Jacobsen, L. K., Lenane M., et al (1998) Multidimensionally impaired disorder: is it a variant of very early-onset schizophrenia? Journal of the American Academy of Child and Adolescent Psychiatry, 37, 91-99.

Lord, C., Rutter, M. L. \& Le Couteur, A. (1994) Autism Diagnostic Interview - Revised: a revised version of a diagnostic interview for caregivers of individuals with possible pervasive developmental disorders. Journal of Autism and Developmental Disorders, 24, 659-685.

__, _-, Dilavore, P. C., et al (1999) Autism Diagnostic Observational Scale (WPS edn). Los Angeles, CA: Western Psychological Services.

Myklebust, H. R. (1975) Non-verbal learning disabilities: Assessment and intervention. In Progress in Learning Disabilities (ed. H. R. Myklebust). New York: Grune and Stratton.

Rapin, I. \& Allen, D. (1983) Developmental language disorders: nosological considerations. In Neuropsychology of Language, Reading, and Spelling (ed. U. Kirk). New York: Academic Press.

Ramsey, J. M., Andresen, N. C. \& Rapoport J. L. (1986) Thought, language, communication and affective flattening in autistic adults. Archives of General Psychiatry, 43, 741-777.

Schmahmann, J. D. \& Sherman, J. C. (1998) The cerebellar cognitive affective syndrome. Brain, 121, 561-579.

Szatmari, P. (1998) Differential diagnosis of Asperger's disorder. In Asperger's Syndrome or High Functioning Autism? (eds E. Schopler, G. Mesibov \& L. Kunce), p. 71 New York: Plenum.

Wing L. (1981) Asperger's syndrome: A clinical account. Psychological Medicine, 11, 115-130.

-- \& Gould, J. (1979) Severe impairments of social interaction and associated abnormalities in children: epidemiology and classification. Journal of Autism and Developmental Disorders, 9, 11-29.

Wolff, S. (1998) Schizoid personality in childhood: the links with Asperger's syndrome, schizophrenia spectrum disorders, and elective mutism. In Asperger's Syndrome or High Functioning Autism (eds E. Schopler, G. Mesibov \& L. Kunce), pp. 123-145. New York: Plenum.

World Health Organization (1992) The ICD-10 Classification of Diseases Clinical Descriptions and Diagnostic Guidelines. Geneva: WHO.

- (1993) The ICD-10 classification of Mental and Behavioural Diosrders: Diagnsotic Criteria for Research. Geneva: WHO.

\section{Multiple choice questions}

1. In Asperger syndrome:

a onset is after age 3 years

b non-verbal deficits are more common than verbal

c motor coordination problems are common

d relatives of affected children have increased risk of schizophrenia

e speech acquisition is normal.

2. DSM-IV criteria for Asperger disorder include:

a lack of enjoyment in activities

b motor mannerisms

c failure to relate to peers

d inability to maintain routines

e lack of social imitative play (appropriate to developmental level).

3. About autism spectrum disorders in adolescence: a they may be misdiagnosed as schizophrenia b features include inattention and overactivity c symptoms become more prominent with age d repetitive activities are resisted

e developmental history is not necessary to make the diagnosis.

4. Asperger syndrome:

a can be clearly differentiated from the autism spectrum disorders

$\mathrm{b}$ is associated with delayed cognitive development

c is more prevalent than classic autism

d has a major genetic component

e resolves in adulthood.

5. Patients with Asperger disorder:

a may meet diagnostic criteria for semantic pragmatic disorder

b are at increased risk of schizophrenia

c are more likely to be boys than girls

$\mathrm{d}$ can be diagnosed with ADHD in DSM-IV

e have an increased risk of mood disorder.

\section{MCQ answers}

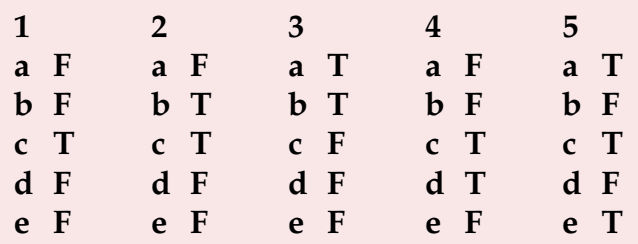

